\title{
Analysis of the efficacy and prognostic factors of PD-1 inhibitors in advanced gallbladder cancer
}

\author{
Qitong Zheng ${ }^{1 \#}$, Chen $\mathrm{Wu}^{1 \#}$, Huangshu $\mathrm{Ye}^{2}$, Zhenggang $\mathrm{Xu}^{1}$, Yang $\mathrm{Ji}^{1}$, Jianhua Rao ${ }^{1}$, Ling Lu ${ }^{1}$, \\ Yaqing Zhu ${ }^{3 *}$, Feng Cheng ${ }^{1 *}$
}

${ }^{1}$ Hepatobiliary Center, First Affiliated Hospital of Nanjing Medical University, Key Laboratory of Liver Transplantation, Research Unit of Liver Transplantation and Transplant Immunology, Chinese Academy of Medical Sciences, Nanjing, China; ${ }^{2}$ Department of Rheumatology, First Affiliated Hospital of Nanjing Medical University, Key Laboratory of Liver Transplantation, Research Unit of Liver Transplantation and Transplant Immunology, Chinese Academy of Medical Sciences, Nanjing, China; ${ }^{3}$ Department of Hepatobiliary Surgery, First Affiliated Hospital of Guangzhou University of Traditional Chinese Medicine, Guangzhou, China

Contributions: (I) Conception and design: Y Zhu, F Cheng; (II) Administrative support: J Rao, L Lu; (III) Provision of study materials or patients: Q Zheng, C Wu; (IV) Collection and assembly of data: Q Zheng, C Wu, H Ye, F Cheng; (V) Data analysis and interpretation: H Ye, Z Xu, Y Ji; (VI) Manuscript writing: All authors; (VII) Final approval of manuscript: All authors.

\#These authors contributed equally to this work.

*These authors contributed equally to this work.

Correspondence to: Feng Cheng. Hepatobiliary Center, First Affiliated Hospital of Nanjing Medical University, Key Laboratory of Liver Transplantation; Research Unit of Liver Transplantation and Transplant Immunology, Chinese Academy of Medical Sciences, Nanjing 210029, China. Email: docchengfeng@njmu.edu.cn; Yaqing Zhu. Department of Hepatobiliary Surgery, the First Affiliated Hospital of Guangzhou University of Traditional Chinese Medicine, 16 Jichang Road, Baiyun District, Guangzhou 510405, China. Email: doctorzhuyq@163.com.

Background: Gallbladder cancer (GBC) is highly malignant, its early diagnosis is difficult, and the 5 -year survival rate is less than 5\%. For patients with advanced GBC (GBCa), combined chemotherapy, radiotherapy, targeted therapy, and immunotherapy are needed to improve the overall survival (OS) rate of patients.

Methods: Data were collected from 53 patients with GBCa who had volunteered to receive programmed death protein-1 (PD-1)-based treatment at the First Affiliated Hospital of Nanjing Medical University from February 2018 to February 2021. Statistical analysis of the collected data, including Kaplan-Meier method, log-rank test, Cox proportional hazard regression model and other methods.

Results: The objective response rates (ORRs) and disease control rates (DCRs) of 53 participants 3 months after receiving immunotherapy were $30.2 \%$ and $79.2 \%$, respectively. The ORRs and DCRs of the combined treatment group were higher than those of the camrelizumab group $(\mathrm{CG})(\mathrm{P}<0.05)$. The DCRs of the camrelizumab plus apatinib group (CAG) at 3 and 6 months were $90.9 \%$ and $45.5 \%(\mathrm{P}=0.003)$, respectively, while the DCRs at 3 and 6 months of the camrelizumab plus chemotherapy group (CCG) were $85.7 \%$ and $71.4 \%(\mathrm{P}=0.450)$, respectively. After treatment, there were statistically significant differences before and after CA199 for each group $(\mathrm{P}<0.05)$. The median progression-free survival (mPFS) of the 53 participants was 7 months, and the median overall survival (mOS) was 12 months. The mPFS and mOS of the CAG and the CCG were greater than those in the CG (6 vs. 3 months, $\mathrm{P}<0.001,12$ vs. 8 months, $\mathrm{P}=0.019 ; 9$ vs. 3 months, $\mathrm{P}<0.001,13$ vs. 8 months, $\mathrm{P}<0.001$, respectively). A total of 16 cases had grade 1 or 2 adverse events, and 3 cases had grade 3 and higher adverse events.

Conclusions: For GBCa patients, PD-1 combined with targeted therapy or chemotherapy is more effective than immunotherapy alone. The targeted therapy group has more obvious early effects on the disease control rate, and combined chemotherapy can achieve sustained effects, providing new ideas for the future GBCa application of immune, targeted, and chemotherapy sequential therapy.

Keywords: Gallbladder cancer (GBC); immunotherapy; immune checkpoint inhibitors (ICIs); targeted therapy; chemotherapy 
Submitted Aug 30, 2021. Accepted for publication Oct 02, 2021.

doi: $10.21037 /$ atm-21-4747

View this article at: https://dx.doi.org/10.21037/atm-21-4747

\section{Introduction}

Gallbladder cancer (GBC) is the most common malignant tumor in the biliary system, mainly situated at the bottom, body, neck, and cystic duct of the gallbladder. Its incidence ranks sixth among gastrointestinal tumors (1), and the bile duct is affected in $0.4-3.8 \%$ of cases. The incidence of GBC varies from country to country. Asia and Latin America have higher incidences, while Europe has relatively low incidences. It is especially common among women (2). Due to the lack of heterogeneous clinical manifestations of GBC, it has a tendency of early lymph node and distant metastases, early diagnosis is difficult, the disease progresses rapidly, and the 5 -year survival rate is less than $5 \%$ (2). Shen et al. (3) showed that the expression of pleckstrin-2 (PLEK2) in GBC tumor tissues is higher than that in noncancerous adjacent tissues. The PLEK2 gene promotes the migration and invasion of GBC cells by regulating the epithelial-mesenchymal transition (EMT) process. It can activate epidermal growth factor receptor (EGFR) signal transduction, chemokines (CC motif) Ligand 2 (CCL2), is the downstream target gene of PLEK2/EGFR signaling, which mediates the movement promotion function of PLEK2.Therefore, the invasion and metastasis of GBC may be related to the EGFR/CCL2 pathway. Radical resection is still the only way to achieve long-term survival. For patients with advanced GBC (GBCa), it is often necessary to combine chemotherapy, radiotherapy, targeted therapy, and immunotherapy to improve the overall survival (OS) rate of patients.

Due to its actions on the tumor microenvironment (TME) (4), immunotherapy can stimulate the body's immune response, mediate the elimination of tumor cells, and achieve antitumor effects. Compared with traditional treatment, immunotherapy is unique in its long-term response and long-term survival in terms of efficacy, unconventional delayed response, and false progress in response mode. Evaluation of the efficacy of immunotherapy is based on the evaluation criteria of immunotherapy for solid tumors. Compared with the traditional approach, measurable new lesions are included in the total tumor burden and compared with the baseline tumor burden (5). Forde et al. (6) found that a large number of $\mathrm{T}$ cells were expanded in peripheral blood and tumor specimens of patients after immunotherapy, indicating that not only a large number of specific $\mathrm{T}$ cells appeared in tumors after immunotherapy, but more importantly, they can enter the blood circulation and reach all parts of the body, including any micrometastasis; because these cells are tumor-specific, they can be eliminated for metastases, thereby effectively reducing postoperative recurrence and metastasis. Among the immunotherapies, immune checkpoint inhibitors (ICIs) are the research focus of immunotherapy, and they have achieved good results in a variety of solid tumors, but the research data on GBC are still lacking. In this study, the clinical data of 53 patients with GBCa were collected and analyzed; these patients were given ICIs to evaluate their effectiveness and safety and provide more patients with safe and effective comprehensive treatment options. We present the following article in accordance with the STROBE reporting checklist (available at https://dx.doi.org/10.21037/ atm-21-4747).

\section{Methods}

\section{Clinical data collection}

Included in the study were patients with GBCa who had volunteered to receive programmed death protein-1 (PD-1)based treatment at the Hepatobiliary Center of the First Affiliated Hospital of Nanjing Medical University from February 2018 to February 2021. Age, gender, performance status (PS), tumor marker CA199 before and after treatment, presence or absence of lymph node metastasis, presence or absence of distant metastasis, treatment plan, surgical status, clinical efficacy, adverse reactions, and disease prognosis were recorded, along with the other clinical characteristics shown in Table 1.

\section{Inclusion and exclusion criteria}

The inclusion criteria were as follows: clear pathological diagnosis and imaging and laboratory examinations; inability to undergo radical resection or recurrence after surgery; at least 1 measurable lesion on imaging; $\geq 18$ years old, ability to provide informed consent; received at least 
Table 1 Clinical features of 53 patients with GBCa

\begin{tabular}{|c|c|}
\hline Clinical features & $\mathrm{N}(\%)$ \\
\hline \multicolumn{2}{|l|}{ Gender } \\
\hline Male & 21 (39.6) \\
\hline Female & $32(60.4)$ \\
\hline \multicolumn{2}{|l|}{ Age (years) } \\
\hline$\geq 60$ & $33(62.3)$ \\
\hline$<60$ & $20(37.7)$ \\
\hline \multicolumn{2}{|l|}{ PS } \\
\hline 0 & $44(83.0)$ \\
\hline 1 & $6(11.3)$ \\
\hline$\geq 2$ & $3(5.7)$ \\
\hline \multicolumn{2}{|l|}{ CA199 (U/mL) } \\
\hline$\geq 65.0$ & $34(64.2)$ \\
\hline$<65.0$ & 19 (35.6) \\
\hline \multicolumn{2}{|l|}{ Lymph node metastasis } \\
\hline Positive & $38(71.7)$ \\
\hline Negative & $15(28.3)$ \\
\hline \multicolumn{2}{|l|}{ Distant metastasis } \\
\hline Yes & $5(9.4)$ \\
\hline No & 48 (90.6) \\
\hline \multicolumn{2}{|l|}{ Treatment options } \\
\hline CG & $10(18.9)$ \\
\hline CAG & $22(41.5)$ \\
\hline CCG & 21 (39.6) \\
\hline \multicolumn{2}{|l|}{ Surgical situation } \\
\hline Postoperative recurrence & $19(35.8)$ \\
\hline Palliative surgery & 21 (39.6) \\
\hline No surgery & $13(24.5)$ \\
\hline
\end{tabular}

In this group of cases, 19 cases had postoperative recurrence; 21 cases underwent palliative surgery, including palliative cholecystectomy, laparoscopic biopsy, bile duct jejunostomy, and other surgical methods; and 13 cases did not undergo surgery. All 53 patients had a clear pathological diagnosis. Pathological specimens were mainly obtained from needle biopsy of liver metastases, palliative surgical biopsy, and laparoscopic biopsy. GBCa, advanced gall bladder cancer; PS, physical fitness score; CG, camrelizumab group; CAG, camrelizumab plus apatinib group; CCG, camrelizumab plus chemotherapy group.
3 cycles of immunosuppressant therapy; survival period $\geq 12$ weeks; no other immunosuppressive agents during treatment; laboratory and imaging data available for 3 and 6 months after treatment. The exclusion criteria were as follows: previous application of other immunotherapy or radiotherapy and interventional therapy; serious heart, brain, lung, kidney, or circulatory system disease; pregnant or breastfeeding; participating in other clinical trials; known or suspected history of allergy to biological agents, severe allergies; history of mental illness; unable to obtain clear pathological results; presence of other malignant tumors or long-term survival with tumors.

\section{Ethical approval}

All procedures performed in this study involving human participants were in accordance with the Declaration of Helsinki (as revised in 2013). This retrospective analysis was approved by the Ethics Committee of the First Affiliated Hospital of Nanjing Medical University (2020-SR-251). All participants were informed of the pros and cons of each treatment option and related risks and adverse events before treatment. Participants and their families signed relevant consent forms. All participants provided informed consent for anti-cancer chemotherapy.

\section{Treatment and grouping}

Participants were allocated to treatment options based on international guidelines and according to their individual situation; participants were informed about treatment options for the disease and the pros and cons of each option before treatment; patients voluntarily selected their treatment plan and signed the relevant consent form. According to the selection plan, 10 cases were allocated to the camrelizumab group (CG), 22 cases to the camrelizumab plus apatinib group (CAG), and 21 cases to the camrelizumab plus chemotherapy group (CCG). As an ICI, camrelizumab was chosen (200 mg, every 3 weeks), and participants received at least 4 cycles of treatment. The targeted drug was apatinib $(250 \mathrm{mg} / \mathrm{d})$, and the chemotherapy drug was gemcitabine combined with oxaliplatin. All treated participants received routine laboratory and imaging examinations, and at least 1 imaging examination every 2 treatment cycles were used to evaluate the treatment effect and record adverse events. 


\section{Efficacy evaluation and adverse events}

The Response Evaluation Criteria in Solid Tumors 1.1 (RECIST) standard was used to evaluate tumor response, including complete response (CR), partial response (PR), stable disease (SD), and progressive disease (PD). Among them, the objective response rates (ORRs) included the number of cases of CR and PR; the disease control rates (DCRs) included the number of cases of CR, PR, and SD. Based on the results, progression-free survival (PFS) and OS were evaluated. The PFS referred to the time from the first treatment to the first disease progression or the death of the participant without any cause, and OS referred to the time from the first treatment to the death of the participant due to any cause. The definition of adverse events was based on the National Cancer Institute Common Terminology Criteria for Adverse Events (CTCAE) version 5.0.

\section{Follow up}

Regular follow ups are carried out by telephone using outpatient information. The start time was the time when the first treatment was received, and the followup deadline was June 2021 During this period, the date of the participant's first disease progression or death were recorded.

\section{Statistical analysis}

All collected data were statistically analyzed using the software SPSS 26.0 (IBM Corp., Armonk, NY, USA). Independent samples $t$-tests were used to analyze continuous variables; chi-square and Fisher's exact tests were used for categorical variables; independent risk factors affecting PFS and OS were analyzed using Cox proportional hazard regression analysis; and the Kaplan-Meier method was used for survival analysis. A P value $<0.05$ was considered to indicate statistical significance.

\section{Results}

A total of 53 cases were enrolled, including 10 cases in the CG, who received treatment for at least 3 cycles; 22 cases in the CAG, who received treatment for at least 3 cycles; and 21 cases in the CCG, who received treatment for at least 3 cycles.

\section{Study population and baseline characteristics}

Of the 53 participants, 21 (39.6\%) were men, and 32
$(60.4 \%)$ were women. The median age overall was 63 years (35-84 years); the median age of males was 62 years (38-80 years), and that of females 63 years (35-84 years). There were $19(35.8 \%)$ cases of recurrence after surgery, 21 (39.6\%) cases underwent palliative surgery, and 13 (24.5\%) cases did not have surgery (Table 1).

\section{Efficacy evaluation}

All 53 participants were treated with camrelizumab, and efficacy was evaluated for the first time after 3 months. Among them, 3, 13, 26, and 11 participants had CR, PR, $\mathrm{SD}$, and PD, respectively, and there were no deaths. The ORRs and DCRs were $30.2 \%$ and $79.2 \%$, respectively. After 6 months, when the curative effect was evaluated again, 0, 3, 22, and 19 participants had CR, PR, SD, and $\mathrm{PD}$, respectively, and 9 had died. The ORRs and DCRs were $5.6 \%$ and $47.2 \%$, respectively. The ORRs of the three groups of participants from the first treatment to the 3 -month follow-up were $0,40.1 \%$, and $33.3 \%$, and the DCRs were $40.0 \%, 90.9 \%$, and $85.7 \%$, respectively. The ORRs and DCRs of the CAG and CCG were better than those of the CG $(\mathrm{P}=0.005)$ (Table 2). When evaluating the efficacy of patients at 3 and 6 months, the ORRs and DCRs of the combined treatment group were significantly different from the CG (Figure 1A,1B). The difference in DCRs between the CAG at 3 and 6 months was statistically significant $(90.9 \%$ vs. $45.5 \%, \mathrm{P}=0.003)$, while the difference in the CCG was not statistically significant $(85.7 \%$ vs. $71.4 \%, \mathrm{P}=0.450$ ) (Figure 1C). In the analysis of the tumor marker CA199 before and after treatment, it was found that the CA199 of each group decreased significantly at the 3-month evaluation (Figure 1D-1F). The CA199 difference of the combined group was statistically significant at the 6-month evaluation (all $\mathrm{P}<0.05$ ) (Figure $1 G, 1 H$ ).

\section{Prognosis and factor analysis}

All 53 participants were followed-up regularly, with a follow-up rate of $100 \%$. As of the time of last follow-up, 23 participants had died. The median PFS (mPFS) of 53 participants was 7 months, and the median OS (mOS) was 12 months. The 3 -month survival rate was $100 \%$, and the 6-month survival rate was $83.0 \%$ (44/53). Univariate analysis of 53 participants showed that age, gender, physical status, CA199, lymph node metastasis, and distant metastasis were not independent risk factors for PFS $(\mathrm{P}>0.05)$, while treatment options were an independent risk 
Table 2 Effect evaluation of 53 patients with GBCa at different times after receiving PD-1 inhibitor treatment [N (\%)]

\begin{tabular}{|c|c|c|c|c|c|c|}
\hline $\begin{array}{l}\text { Time and } \\
\text { grouping }\end{array}$ & \multicolumn{6}{|c|}{ Efficacy evaluation } \\
\hline 3 months & $3(5.6)$ & $13(24.5)$ & $26(49.1)$ & $11(20.8)$ & $16(30.2)$ & $42(79.2)$ \\
\hline 6 months & 0 & $3(5.6)$ & $22(41.5)$ & 19 (35.8) & $3(5.6)$ & $25(47.2)$ \\
\hline CAG $(n=22)$ & $1(4.5)$ & $8(36.4)$ & $11(50.0)$ & $2(9.1)^{*}$ & $9(40.1)^{\star}$ & $20(90.9)^{\star}$ \\
\hline CCG $(n=21)$ & $2(9.5)$ & $5(23.8)$ & $11(52.4)$ & $3(14.3)^{*}$ & $7(33.3)^{\star}$ & $18(85.7)^{\star}$ \\
\hline
\end{tabular}

*, compared with the camrelizumab group alone, P<0.05. GBCa, advanced gall bladder cancer; CG, camrelizumab group; CAG, camrelizumab plus apatinib group; CCG, camrelizumab plus chemotherapy group; CR, complete remission; PR, partial remission; SD, stable disease; PD, disease progression; ORR, objective response rate; $\mathrm{DCR}$, disease control rate.

factor for PFS [hazard ratio (HR): $0.238,95 \%$ CI: 0.102 to $0.556, \mathrm{P}=0.001$; HR: $0.131,95 \% \mathrm{CI}: 0.053$ to 0.326 , $\mathrm{P}=0.001]$. The single factor $\mathrm{P}<0.1$ variable was included in the multivariate analysis, and it was found that the tumor marker CA199 (HR: 2.420, 95\% CI: 1.294 to 4.528 , $\mathrm{P}=0.006)$ and treatment options (HR: 0.264, 95\% CI: 0.106 to $0.661, \mathrm{P}=0.004$; HR: $0.103,95 \% \mathrm{CI}: 0.039$ to 0.276 , $\mathrm{P}=0.001$ ) were independent risk factors for PFS (Table 3). Multivariate analysis showed that distant metastasis (HR: $3.753,95 \%$ CI: 1.261 to $11.172, \mathrm{P}=0.017)$ and treatment options (HR: $0.274,95 \%$ CI: 0.078 to $0.968, \mathrm{P}=0.044$; HR: $0.204,95 \%$ CI: 0.035 to $0.516, \mathrm{P}=0.003$ ) were independent risk factors for OS, while age, gender, CA199, physical status, and lymph node metastasis were not risk factors $(\mathrm{P}>0.05)$ (Table 4).

\section{Subsistence analysis}

The mPFS of the 3 groups of participants was 3, 6, and 9 months, and the mOS was 8,12 , and 13 months, respectively. The mPFS and mOS of the combination group were better than those of the CG $(\mathrm{P}<0.05)$. However, when the mPFS and mOS of the CCG were compared with the CAG (9 vs. 7 months, $\mathrm{P}=0.029$, 13 vs. 12 months, $\mathrm{P}=0.10$ ), the mPFS difference was statistically significant, while the mOS difference was not statistically significant (Figures 2,3 ).

\section{Adverse events}

The overall incidence of adverse events in 53 participants was $47.2 \%$ (25/53), and the incidence of immune-related adverse events (irAE) was 35.8\% (19/53). Grade 1 and Grade 2 adverse events accounted for the majority, including dizziness, fatigue, thrombocytopenia, and head, face, and body rash; Grade 3 or 4 adverse events occurred in $5.7 \%$ (3/53), mainly related to immune-related inflammation, including hypothyroidism, myocarditis, and nephritis, all of which can improve with active treatment.

\section{Discussion}

The invasive ability of GBC is strong, it has a high degree of malignancy, and a poor prognosis. Most patients no longer have the option of radical resection by the time of diagnosis. Therefore, patients with GBCa urgently need comprehensive anti-tumor treatment. In order to improve the overall treatment effect of GBC, it is crucial to swiftly implement the concept of comprehensive treatment based on standardized radical resection, combined with chemotherapy, radiotherapy, targeted therapy, and immunotherapy. Surgical methods, tumor location, distant metastasis, tumor-node-metastasis (TNM) staging, tumor pathological type, and tumor differentiation are the main factors affecting the prognosis and recurrence of GBC. Valle et al. (7) have shown that for patients with advanced biliary malignancies, the standard first-line treatment is gemcitabine combined with cisplatin. However, studies such as that by Azizi et al. (8) found that gemcitabine combined with cisplatin increases ORR and DCR when treating GBCa, but has no obvious effect on improving PFS and OS. Therefore, there is an urgent need for improved combined treatment options to ameliorate patient prognosis. Topalian 

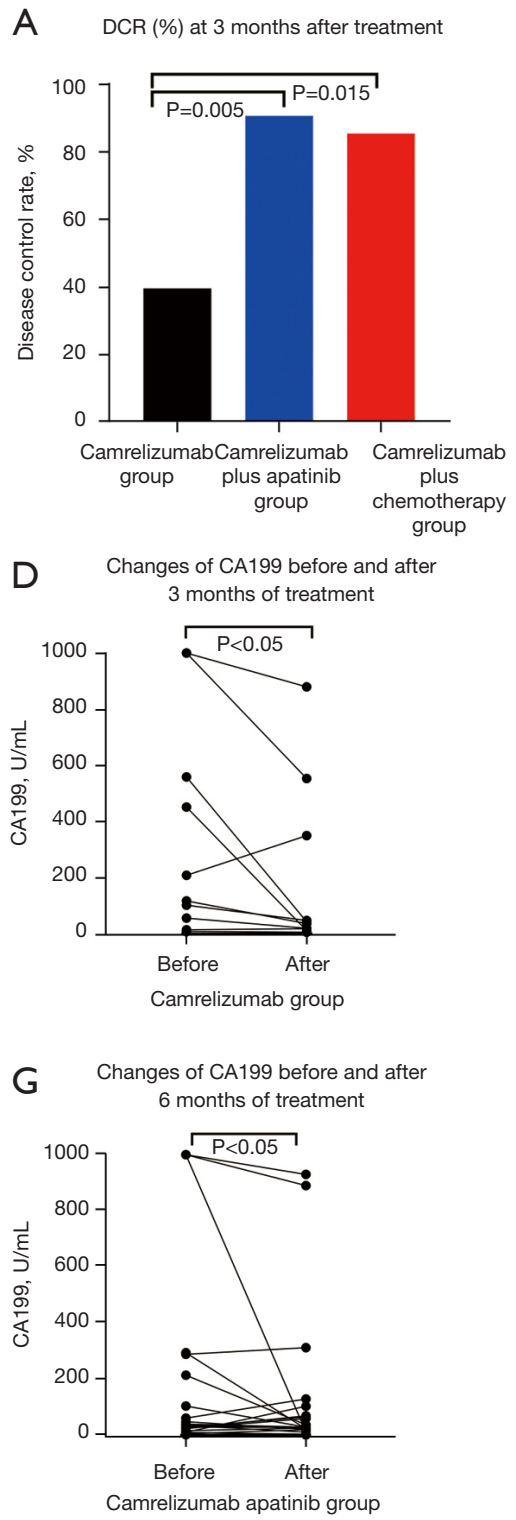

B

DCR (\%) at 6 months after treatment

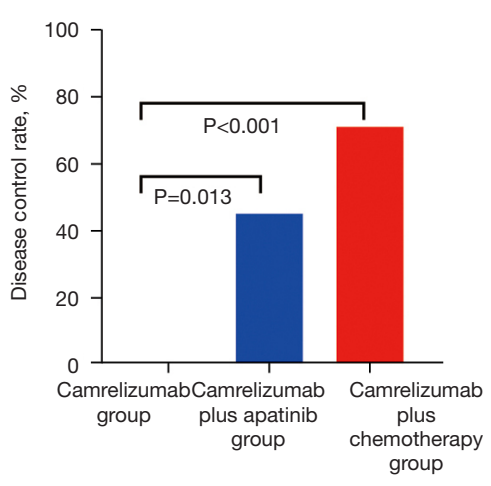

E
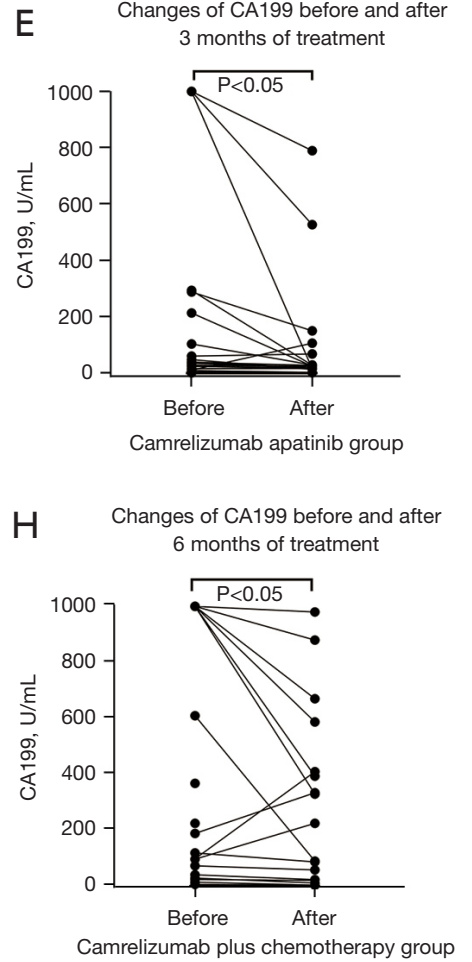

C Changes in DCR (\%) for 3 months and 6 months of treatment

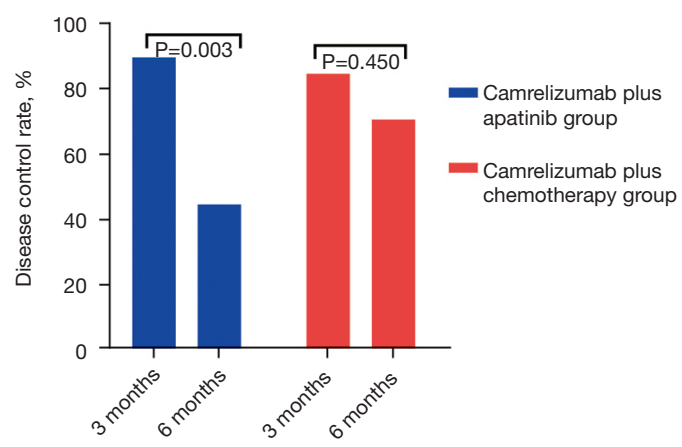

$\mathrm{F}$

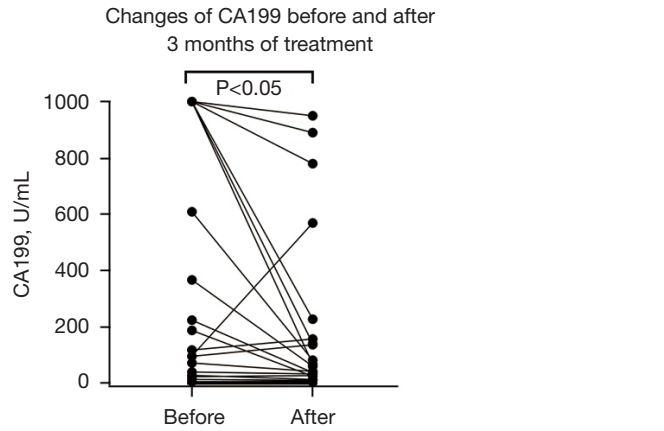

Camrelizumab plus chemotherapy group

Figure 1 Changes of DCR and CA199 before and after treatment in the 3 groups. (A) Disease control rates of 3 groups of patients when the curative effect was evaluated at 3 months; (B) disease control rates of the 3 groups of patients at 6 months of curative effect evaluation; (C) comparison of disease control rates between 3 months and 6 months in the combined group; (D) changes before and after CA199 in the camrelizumab group at 3 months; (E) changes in CA199 before and after 3 months in the camrelizumab plus apatinib group; (F) changes in CA199 before and after 3 months in the camrelizumab plus chemotherapy group; $(\mathrm{G})$ changes in CA199 before and after 6 months in the camrelizumab plus apatinib group; (H) changes in CA199 before and after 6 months in the camrelizumab plus chemotherapy group. DCR, disease control rate.

et al. (9) showed that about $20-25 \%$ of patients with antiPD-1 antibody treatment have an objective effect, and adverse drug reactions are safe and controllable. It has been found that $36 \%$ of PD-L1 positive patients have an objective remission, while among PD-L1 negative patients, no objective remission was observed, which indicates that there is a certain correlation between the expression of PD-L1 on the cell surface of the tumor before standard treatment and the objective therapeutic effect. However, it is necessary to use PD-L1 as a potential molecular marker 
Table 3 Univariate and multivariate analysis of progression-free survival correlations in 53 patients using a Cox proportional hazard model

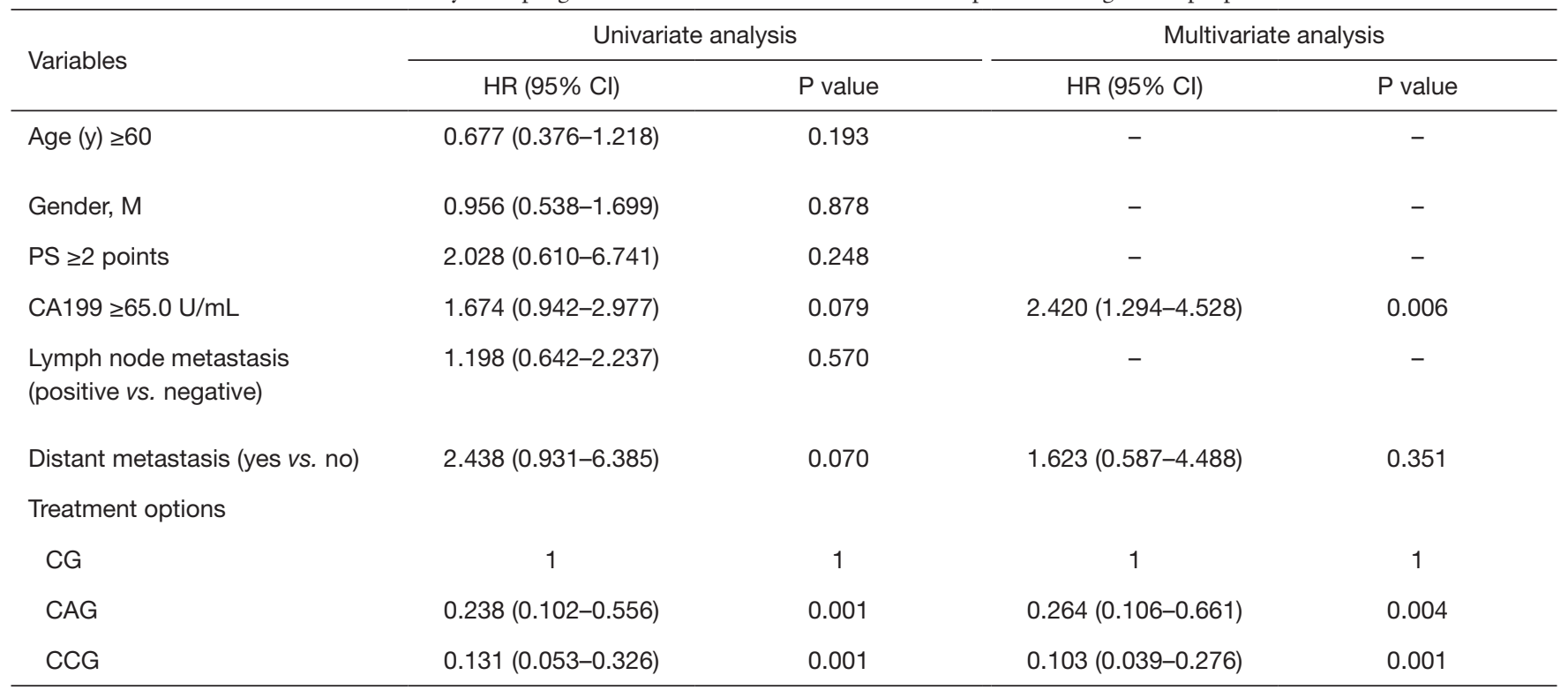

PS, physical fitness score; CG, camrelizumab group; CAG, camrelizumab plus apatinib group; CCG, camrelizumab plus chemotherapy group; HR, hazard ratio; $\mathrm{Cl}$, confidence interval.

Table 4 Univariate and multivariate analysis of overall survival correlations in 53 patients using a Cox proportional hazard model

\begin{tabular}{|c|c|c|c|c|}
\hline Variables & \multicolumn{2}{|c|}{ Univariate analysis } & \multicolumn{2}{|c|}{ Multivariate analysis } \\
\hline Age $(y) \geq 60$ & $1.411(0.594-3.354)$ & 0.435 & - & - \\
\hline Gender, M & $0.647(0.298-1.405)$ & 0.271 & - & - \\
\hline$P S \geq 2$ points & $2.852(0.844-9.635)$ & 0.092 & $2.104(0.575-7.707)$ & 0.261 \\
\hline $\begin{array}{l}\text { Lymph node metastasis } \\
\text { (positive vs. negative) }\end{array}$ & $1.497(0.654-3.429)$ & 0.340 & - & - \\
\hline Distant metastasis (yes vs. no) & $3.716(1.352-10.213)$ & 0.011 & $3.753(1.261-11.172)$ & 0.017 \\
\hline \multicolumn{5}{|l|}{ Treatment options } \\
\hline
\end{tabular}

PS, physical fitness score; CG, camrelizumab group; CAG, camrelizumab plus apatinib group; CCG, camrelizumab plus chemotherapy group; HR, hazard ratio; $\mathrm{Cl}$, confidence interval.

to predict anti-PD-1 antibody treatment, and the effect of this approach requires further research to confirm. In advanced tumors such as lung cancer, melanoma, and liver cancer (10), immunotherapy has achieved good results, providing a new direction for future comprehensive treatments. This study retrospectively analyzed 53 patients with GBCa who received immunotherapy, among whom, 10 participants received camrelizumab alone, 22 received camrelizumab plus apatinib therapy, and 21 received camrelizumab plus chemotherapy. The whole 53 participant cohort was evaluated for the first time after 3 months, and the ORRs and DCRs were $30.2 \%$ and $79.2 \%$, respectively. 


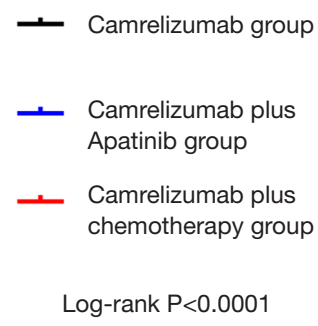

Number at risk

Camrelizumab group Camrelizumab plus apatinib group Camrelizumab plus chemotherapy group

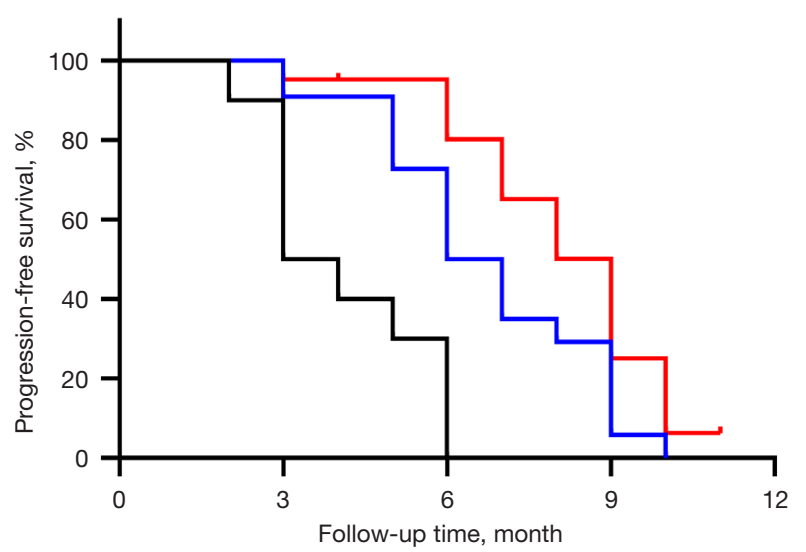

$\begin{array}{rrrrr}10 & 9 & 3 & 0 & 0 \\ 22 & 22 & 16 & 5 & 0 \\ 21 & 21 & 19 & 10 & 0\end{array}$

Figure 2 The progression-free survival curves of the three groups of patients.

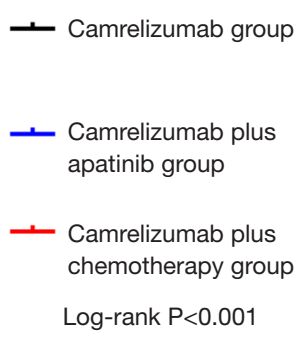

Number at risk Camrelizumab group

Camrelizumab plus apatinib group

Camrelizumab plus chemotherapy group

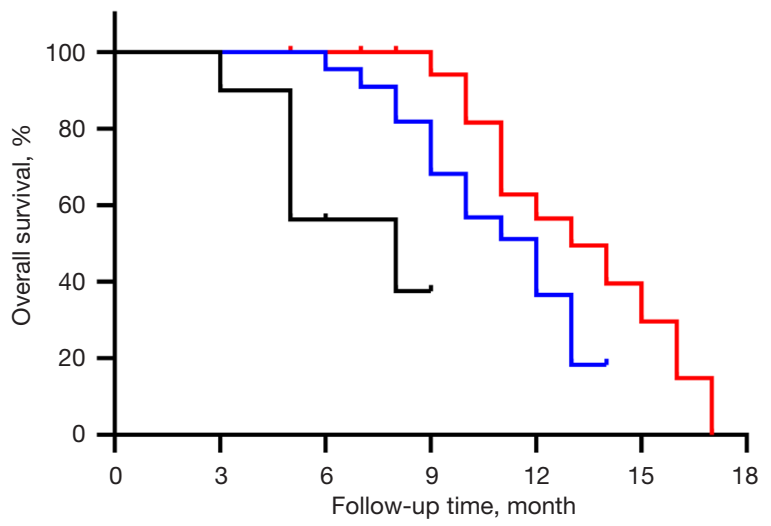

$\begin{array}{rrrrrrr}10 & 10 & 4 & 2 & 0 & 0 & 0 \\ 22 & 22 & 22 & 18 & 7 & 0 & 0 \\ 21 & 21 & 20 & 17 & 10 & 4 & 0\end{array}$

Figure 3 The overall survival curve of the 3 groups of patients.

The ORRs of the 3 groups of participants were $0,40.1 \%$, and $33.3 \%$, and the DCRs were $40.0 \%, 90.9 \%$, and $85.7 \%$, respectively. The ORRs and DCRs at 3 and 6 months in the CAG were $90.9 \%$ and $45.5 \%$, respectively, and the ORRs and DCRs at 3 and 6 months in the CCG were $85.7 \%$ and $71.4 \%$, respectively. The CA209-538 phase II (11) clinical trial mainly assessed the effectiveness and safety of combined immunotherapy for advanced biliary tract cancer. Among them, the ORR of GBC was $31 \%$. The application of immunotherapy in patients with $\mathrm{GBCa}$ for disease control underscores previous studies. The combination therapy group performed better than the single-agent group in terms of disease control, which to a certain extent shows that combination therapy can lead to a better patient prognosis. When reassessed 6 months later, it was found that there was no difference in DCR changes in the CCG, while the difference in DCR changes in the CAG was statistically significant. Merla et al. (12) showed that targeted therapy in the short-term can have a significant effect in eliminating susceptible tumors. Therefore, the results of this study suggest to a certain extent that the targeted therapy group had more obvious early effects, and 
combined chemotherapy can achieve a continuous effect, showing great potential for the sequential treatment of immune, targeted, and chemotherapy for patients with GBCa.

The mPFS and mOS of the 53 participants were 7 and 12 months, respectively. Compared with first-line chemotherapy, immunotherapy has more advantages. In a single-arm phase II clinical trial (13), the proportion of GBCa was $44 \%$, and the DCR at 3 months for targeted therapy was $48 \%$. Syed Khaja et al. (14) found that targeted drugs can attenuate tumor-induced immune suppression, improve immune response, and strengthen the persistence of immune efficacy. In this study, the mPFS and mOS of the CAG were 6 and 12 months compared to the CG with 3 and 8 months, respectively, confirming that combined immune targeting is a valuable treatment option. A single-arm clinical trial (15) showed that, when the enrolled advanced cholangiocarcinoma and GBC patients were treated with immunotherapy combined with chemotherapy, 12 cases of CR and 12 cases of SD achieved good results. The JAPICCTI-153098 phase I (13) trial is a series of studies exploring nivolumab as monotherapy or combination chemotherapy in biliary tract cancer. The mPFS and mOS of monotherapy and combination therapy were 1.4 and 5.2 months and 4.2 and 15.4 months, respectively. In this study, mPFS and mOS in the CCG were 9 months and 13 months and hence longer than those of the CG. Research (16) suggests that in addition to increasing the release of antigen when eliminating tumor cells, chemotherapeutic drugs can also change the tumor microenvironment and promote antigen expression, thereby reactivating the immune response. In this study, it was confirmed that combined chemotherapy can exert its synergistic effect to achieve a better prognosis. Wang et al. (17) showed that the expression level of PD-L1 in advanced biliary malignant tumors has no correlation with different ICIs and PFS, while treatment options such as ICIs combined with chemotherapy or targeted therapy are independently related to PFS. This study did not focus on individual comparisons of advanced GBC, but concentrated on the overall advanced biliary malignancy. This article does not specifically detect PD-L1 in patients, which is a bit regretful; in the following clinical work, we need to continue to explore the efficacy of its expression level in patients with GBCa, and observe the effects of different drug types.

In addition, Kim et al. (18) showed that the tumor marker CA199 $\geq 65 \mathrm{U} / \mathrm{mL}$ is an important prognostic factor in patients with GBCa. Chen et al. (19) proposed that the specificity and sensitivity of CA199 in the diagnosis of advanced GBC were $89.7 \%$ and $89 \%$, respectively. In the evaluation of the treatment effect of patients, the level of CA199 is closely related to the treatment effect. Haring et al. (20) found that inflammation of the gallbladder or bile duct will increase CA199, and cholecystectomy will also affect its expression. In this study, the 53 participants showed statistically significant differences in CA199 before and after treatment. In multivariate Cox regressions, CA199 was an independent risk factor in PFS, but it was not an independent risk factor in OS. In some patients, this may be related to postoperative recurrence or palliative resection, and interference with the bile duct may cause false positive increases. Similar to targeted drugs for precise treatment, precise screening of patients with high immune responses will be a significant focus of antitumor therapy in the future. In recent years, studies have shown that the high expression of the intratumoral biomarker PD-1, high tumor mutation load, and high microsatellite instability are better for immunotherapy $(21,22)$, and that the extraneous biomarker peripheral blood CD8+ T cells activation and circulating tumor DNA can reflect tumor burden and predict treatment response $(23,24)$. Early diagnosis of GBC is difficult, and it is necessary to find new diagnostic and prognostic markers. In recent years, single-cell RNAseq technology has matured. It extracts total RNA from GBC and adjacent tissues, and builds a database after amplification, then performs transcriptome sequencing, and correlation analysis is conducted based on patient clinical information. The survivin, TK1, TNNT1, and MMP9 genes have been clinically found to be related to prognosis. These differentially expressed genes are potential new indicators for the diagnosis and prognosis of GBC (25). In this study, we did not perform detailed tumor genetic testing and peripheral blood analysis on the 53 patients included. Therefore, to benefit more patients in the future, precision immunotherapy is essential.

With the advent of immunotherapy, irAE began to receive increased attention. Studies have shown (26) that its main adverse reactions are fever, fatigue, rash, pruritus, pneumonia, hypothyroidism, liver damage, immune myocarditis, enteritis, and nephritis. Also, about $49 \%$ of patients experience any level of irAEs, which can be expressed in any tissue and organ, but mainly occur in the skin, endocrine organs, heart, liver, and kidney. Clinical studies have shown that the incidence of irAE is $60-80 \%(27,28)$. The incidence of irAEs of different 
immunosuppressive agents varies, and the incidence of irAEs of immune combination therapy is higher than that of immune monotherapy (29). Khoja et al. (30) showed that when receiving PD-1 inhibitor treatment, different tumors have different irAE characteristics. Due to the differences in anti-tumor immune responses among patients with different tumor types receiving the same ICI treatment; tumor microenvironment, immune penetration, adaptive immune response, and the formation of neoantigens contribute to histological specific irAE patterns. As more data from the ICI trial becomes available, other tumor-dependent characteristics of irAE will be identified. The incidence of irAE in this study was $35.8 \%$ lower than that reported in the literature, which may overlap with the adverse reactions caused by combined targeting and chemotherapy in the treatment plan, and these adverse reactions may be difficult to distinguish. When administering combined targeted therapy, attention should be paid to cardiovascular and cerebrovascular problems, early detection, and early treatment; when administering combined chemotherapy treatment, attention should be paid to bone marrow suppression and endocrine imbalance. Most of the related adverse reactions improve after active treatment. Whether there is a correlation between the incidence and severity of irAE and the efficacy of immunotherapy is controversial. No reliable conclusions can be drawn as yet, and a large number of clinical studies are needed to confirm a potential correlation $(31,32)$.

\section{Conclusions}

Based on the results of this study, immunotherapy can offer hope to patients with GBCa. Combination therapy can increase the response rate of immunotherapy and improve its therapeutic effect. In particular, combined chemotherapy can significantly prolong the PFS and OS of patients. In addition, this study found that in terms of short-term disease control, targeted therapy has an advantage over chemotherapy, but chemotherapy is more active in terms of sustained therapeutic effects. Therefore, in the future, the sequential treatment of immune, targeted, and chemotherapy for patients with GBCa is a potentially valuable solution.

\section{Acknowledgments}

Funding: The project was supported by the National Natural Science Foundation of China (81871259 and
820706752), and the Chinese Academy of Medical Sciences Innovation Fund for Medical Sciences (2019-I2M-5-035).

\section{Footnote}

Reporting Checklist: The authors have completed the STROBE reporting checklist. Available at https://dx.doi. org/10.21037/atm-21-4747

Data Sharing Statement: Available at https://dx.doi. org/10.21037/atm-21-4747

Conflicts of Interest: All authors have completed the ICMJE uniform disclosure form (available at https://dx.doi. org/10.21037/atm-21-4747). All authors reported that the project was supported by the National Natural Science Foundation of China (81871259 and 820706752), and the Chinese Academy of Medical Sciences Innovation Fund for Medical Sciences (2019-I2M-5-035). Dr. LL serves as an unpaid Associate Editors-in-Chief of Annals of Translational Medicine from Jun 2019 to May 2024. The authors have no other conflicts of interest to declare.

Ethical Statement: The authors are accountable for all aspects of the work in ensuring that questions related to the accuracy or integrity of any part of the work are appropriately investigated and resolved. All procedures performed in this study involving human participants were in accordance with the Declaration of Helsinki (as revised in 2013). The study was approved by the Ethics Committee of the First Affiliated Hospital of Nanjing Medical University (Number: 2020-SR-251), and participants and their families signed relevant consent forms.

Open Access Statement: This is an Open Access article distributed in accordance with the Creative Commons Attribution-NonCommercial-NoDerivs 4.0 International License (CC BY-NC-ND 4.0), which permits the noncommercial replication and distribution of the article with the strict proviso that no changes or edits are made and the original work is properly cited (including links to both the formal publication through the relevant DOI and the license). See: https://creativecommons.org/licenses/by-nc-nd/4.0/.

\section{References}

1. Hundal R, Shaffer EA. Gallbladder cancer: epidemiology and outcome. Clin Epidemiol 2014;6:99-109. 
2. Goetze TO. Gallbladder carcinoma: Prognostic factors and therapeutic options. World J Gastroenterol 2015;21:12211-7.

3. Shen H, He M, Lin R, et al. PLEKpromotes gallbladder cancer invasion and metastasis through EGFR/CCL2 pathway. J Exp Clin Cancer Res 2019;38:247.

4. Christofi T, Baritaki S, Falzone L, et al. Current Perspectives in Cancer Immunotherapy. Cancers (Basel) 2019;11:1472.

5. Seymour L, Bogaerts J, Perrone A, et al. iRECIST: guidelines for response criteria for use in trials testing immunotherapeutics. Lancet Oncol 2017;18:e143-52.

6. Forde PM, Chaft JE, Smith KN, et al. Neoadjuvant PDBlockade in Resectable Lung Cancer. N Engl J Med 2018;378:1976-86.

7. Valle J, Wasan H, Palmer DH, et al. Cisplatin plus gemcitabine versus gemcitabine for biliary tract cancer. $\mathrm{N}$ Engl J Med 2010;362:1273-81.

8. Azizi AA, Lamarca A, McNamara MG, et al. Chemotherapy for advanced gallbladder cancer (GBC): A systematic review and meta-analysis. Crit Rev Oncol Hematol 2021;163:103328.

9. Topalian SL, Hodi FS, Brahmer JR, et al. Safety, activity, and immune correlates of anti-PD-1 antibody in cancer. $\mathrm{N}$ Engl J Med 2012;366:2443-54.

10. Finn RS, Qin S, Ikeda M, et al. Atezolizumab plus Bevacizumab in Unresectable Hepatocellular Carcinoma. N Engl J Med 2020;382:1894-905.

11. Klein O, Kee D, Nagrial A, et al. Evaluation of Combination Nivolumab and Ipilimumab Immunotherapy in Patients With Advanced Biliary Tract Cancers: Subgroup Analysis of a Phase 2 Nonrandomized Clinical Trial. JAMA Oncol 2020;6:1405-9.

12. Merla A, Liu KG, Rajdev L. Targeted Therapy in Biliary Tract Cancers. Curr Treat Options Oncol 2015;16:48.

13. Ueno M, Ikeda M, Morizane C, et al. Nivolumab alone or in combination with cisplatin plus gemcitabine in Japanese patients with unresectable or recurrent biliary tract cancer: a non-randomised, multicentre, open-label, phase study. Lancet Gastroenterol Hepatol 2019;4:611-21.

14. Syed Khaja AS, Toor SM, El Salhat H, et al. Preferential accumulation of regulatory $T$ cells with highly immunosuppressive characteristics in breast tumor microenvironment. Oncotarget 2017;8:33159-71.

15. Chen $\mathrm{X}, \mathrm{Wu} \mathrm{X}, \mathrm{Wu} \mathrm{H}$, et al. Camrelizumab plus gemcitabine and oxaliplatin (GEMOX) in patients with advanced biliary tract cancer: a single-arm, open-label, phase II trial. J Immunother Cancer 2020;8:e001240.

16. Zitvogel L, Galluzzi L, Smyth MJ, et al. Mechanism of action of conventional and targeted anticancer therapies: reinstating immunosurveillance. Immunity 2013;39:74-88.

17. Wang JH, Li W, Ma WC, et al. Efficacy and prognostic factors of programmed cell death protein-inhibitors in the treatment of advanced biliary tract cancer. Chinese Journal of Practical Surgery 2021;41:559-64.

18. Kim M, Kim H, Han Y, et al. Prognostic Value of Carcinoembryonic Antigen (CEA) and Carbohydrate Antigen 19-9 (CA 19-9) in Gallbladder Cancer; 65 IU/ mL of CA 19-9 Is the New Cut-Off Value for Prognosis. Cancers (Basel) 2021;13:1089.

19. Chen Z, Liu Z, Zhang Y, et al. Combination of CA19-

9 and the Neutrophil-to-Lymphocyte Ratio for the Differential Diagnosis of Gallbladder Carcinoma. Cancer Manag Res 2020;12:4475-82.

20. Haring MPD, de Cort BA, Nieuwenhuijs VB. Elevated CA19-9 levels; not always cancer. Ned Tijdschr Geneeskd 2021;164:D4048.

21. Arkenau HT, Martin-Liberal J, Calvo E, et al. Ramucirumab Plus Pembrolizumab in Patients with Previously Treated Advanced or Metastatic Biliary Tract Cancer: Nonrandomized, Open-Label, Phase I Trial (JVDF). Oncologist;23:1407-e136.

22. Chen J, Quan M, Chen Z, et al. Camrelizumab in advanced or metastatic solid tumour patients with DNA mismatch repair deficient or microsatellite instability high: an open-label prospective pivotal trial. J Cancer Res Clin Oncol 2020;146:2651-7.

23. Peng G, Li S, Wu W, et al. PD-1 upregulation is associated with $\mathrm{HBV}$-specific $\mathrm{T}$ cell dysfunction in chronic hepatitis B patients. Mol Immunol 2008;45:963-70.

24. Wu X, Li J, Gassa A, et al. Circulating tumor DNA as an emerging liquid biopsy biomarker for early diagnosis and therapeutic monitoring in hepatocellular carcinoma. Int J Biol Sci 2020;16:1551-62.

25. Gu X, Jiang MM, Gao CF, et al. Screening new diagnostic and prognostic markers for gallbladder cancer using RNAseq technology. Compilation of the National Conference on Immunology 2015:273-4.

26. Xie C, Duffy AG, Mabry-Hrones D, et al. Tremelimumab in Combination With Microwave Ablation in Patients With Refractory Biliary Tract Cancer. Hepatology 2019;69:2048-60.

27. Hodi FS, O'Day SJ, McDermott DF, et al. Improved survival with ipilimumab in patients with metastatic melanoma. N Engl J Med 2010;363:711-23. 
28. Herbst RS, Baas P, Kim DW, et al. Pembrolizumab versus docetaxel for previously treated, PD-L1-positive, advanced non-small-cell lung cancer (KEYNOTE-010): a randomised controlled trial. Lancet 2016;387:1540-50.

29. Arnaud-Coffin P, Maillet D, Gan HK, et al. A systematic review of adverse events in randomized trials assessing immune checkpoint inhibitors. Int J Cancer 2019;145:639-48.

30. Khoja L, Day D, Wei-Wu Chen T, et al. Tumour- and class-specific patterns of immune-related adverse events of immune checkpoint inhibitors: a systematic review. Ann Oncol 2017;28:2377-85.

Cite this article as: Zheng Q, Wu C, Ye H, Xu Z, Ji Y, Rao J, Lu L, Zhu Y, Cheng F. Analysis of the efficacy and prognostic factors of PD-1 inhibitors in advanced gallbladder cancer. Ann Transl Med 2021;9(20):1568. doi: 10.21037/atm-21-4747
31. Downey SG, Klapper JA, Smith FO, et al. Prognostic factors related to clinical response in patients with metastatic melanoma treated by CTL-associated antigen-4 blockade. Clin Cancer Res 2007;13:6681-8.

32. Horvat TZ, Adel NG, Dang TO, et al. Immune-Related Adverse Events, Need for Systemic Immunosuppression, and Effects on Survival and Time to Treatment Failure in Patients With Melanoma Treated With Ipilimumab at Memorial Sloan Kettering Cancer Center. J Clin Oncol 2015;33:3193-8.

(English Language Editors: B. Meiser and J. Jones) 